\title{
Associations of HLA DRB1 Alleles with Igg Oligoclonal Bands and Their Influence on Multiple Sclerosis Course and Disability Status
}

Renata Balnyte*, Daiva Rastenyte, Antanas Vaitkus, Ingrida Uloziene, Astra Vitkauskiene and Erika Skrodeniene

Department of Neurology, LUHC Kaunas Clinics, Kaunas, Lithuania

*Corresponding author: Renata BalnyteNeurologist, LUHC Kaunas Clinics, Neurology, Eiveniu, Kaunas, Lithuania, E-mail: balnyter@hotmail.com

Received date: Dec 14, 2014, Accepted date: Feb 20, 2015, Published date: Feb 24, 2015

Copyright: (c) 2015 Balnyte R, et al. This is an open-access article distributed under the terms of the Creative Commons Attribution License, which permits unrestricted use, distribution, and reproduction in any medium, provided the original author and source are credited.

\begin{abstract}
Background: Oligoclonal bands (OCB) may be associated with the genes of HLA complex, which allows to consider the possible interaction of genetic and immunological factors and its importance in the development and progression of multiple sclerosis (MS).

Aim: To evaluate the importance of the associations between HLA DRB1 alleles and oligoclonal bands (OCB) in the multiple sclerosis (MS) patients disease course and disability.

Metods: This was a prospective study of 120 patients with MS. HLA DRB1 alleles were genotyped using the polymerase chain reaction. Matched cerebrospinal fluid (CSF) and plasma samples were analysed using isoelectric focusing and IgG specific immunofixation to test for the presence of intrathecal specific OCB and compared directly with the serum samples.

Results: HLA DRB1*08 allele be related to lower degree of disability, while the other HLA DRB1 alleles irrespective of the presence or absence of OCBs did not influence disability status. Oligoclonal bands were an indepedent and significant factor that influenced disability status irrespective of HLA DRB1* 04, *07, *08, *, *13, *15 and ${ }^{*} 16$ alleles. Age at the onset of the first symptoms and duration of the disease were independent and significant factors for MS progression in all logistic regression models with each newly added HLA DRB1 allele. HLA DRB1*08 allele was related to $75 \%$ lower odds that relapsing remitting (RR) MS will change to a progressive course MS irrespective of the other factors investigated. In the model with *08 allele immunological factors had no impact on MS progression. Detection of OCBs in the CF was associated with the higher possibility of RR MS progression in all cases, except then the *08 allele was present.
\end{abstract}

Conclusions: OCBs had an influence on disability status, while HLA DRB $1^{*} 08$ allele was significantly associated with lower possibility that RR MS will change to progressive course MS.

Keywords: Oligoclonal bands; HLA DRB1 allele; EDSS, Disability; Multiple sclerosis

\section{Background}

Multiple sclerosis (MS) is the most common and severe idiopathic demyelinating disease of the central nervous system with etiopathogenesis involving a complex interaction between genetic and environmental factors [1-3]. Epidemiological data support a view that MS is caused or triggered by some environmental factors in persons who are genetically susceptible [4]. It was demonstrated that the HLA complex is not only associated with the disease development, but can also influence certain clinical features and immunological changes in the patients with MS $[3,4]$. The interdependence of HLA-DRB1 genotype and the phenotypic status of oligoclonal bands have been suggested [5]. Higher prevalence of HLA-DRB1 ${ }^{*} 1501$ allele may be linked to an earlier onset of the disease, less favourable course and female gender, the factors which are believed to be particularly important in the prognosis and treatment of MS [6,7]. Oligoclonal immunoglobulin G (IgG) bands (OCBs) are present in the cerebrospinal fluid (CSF) of the majority (85 - 95\%) of clinically definite MS patients and are believed to reflect the intrathecal synthesis of IgG antibodies [8,9]. OCBs are detected in $76-97 \%$ Western Europeans with MS [10-12] and 55 - 56\% MS patients in Asian countries [13-16]. These differences may be related to the both geographical location and ethnic features, as well as immunogenetic factors, that influence intrathecal humoral immune function [11-17]. Opinions about OCBs impact on MS course are controversial detection of OCBs could be associated with more aggressive course of the disease [13,18-21], however, according to other sources, OCBs are related to slower MS progression, better course and lower disability level, as well as female sex [14].

The aim of the present study was to determine the relationship of immunogenetic risk factors with the clinical features and disability status of MS patients in Lithuanian population.

\section{Material and Methods}

\section{Subjects}

The 120 MS patients, older than 18 years, referred to the Department of Neurology at the Hospital of Lithuanian University of Health Sciences in Kaunas during 2009- 2010 and willing to 
Citation: Balnyte R, Rastenyte D, Vaitkus A, Uloziene I, Vitkauskiene A, et al. (2015) Associations of HLA DRB1 Alleles with Igg Oligoclonal Bands and Their Influence on Multiple Sclerosis Course and Disability Status. 2155-9562 6: 273. doi:10.4172/2155-9562.1000273

Page 2 of 5

participate into the study composed the study group. The study included patients with confirmed MS diagnosis only. MS diagnosis was established according to widely accepted and revised McDonald criteria (2005) [22]. Lumbar puncture and cerebrospinal fluid (CSF) examination was performed at the time of diagnosis.

Matched CSF and plasma samples were analysed using isoelectric focusing and IgG specific immunofixation to test for the presence of intrathecal specific OCB and compared directly with the serum samples [18]. Positive OCBs were defined as more than 2 bands present in the CSF, but absent in the corresponding blood serum [8].

Demographic (age at onset of the first symptoms, gender) and clinical data (disease course and duration of the symptoms, disability status), the results of all paraclinical tests were collected for all patients. Disability was measured using the Kurtzke Expanded Disability Status Scale (EDSS) scores. The patients were followed up prospectively and their clinical status was checked every three months.

The study was approved by the Kaunas Regional Bioethics Committee [No 2R-493]. Informed consent form was signed by each patient before the entering the study.

HLA genotyping: Blood samples were obtained from the patients with $\mathrm{MS}$ and stored at $-20^{\circ} \mathrm{C}$. DNA was extracted from blood leukocytes by standard phenol-chloroform method. DNA was dissolved in the sterile double distilled water. HLA DRB1 alleles for MS patients were genotyped using a polymerase chain reaction (PCR) with amplification of the second exon of the genes. An amplified product was manually dot blotted onto nylon membranes. Synthetic sequence-specific oligonucleotide probes were 3 '-end- labelled with aP32-dCTP and used for hybridization followed by stringency washes and autoradiography. HLA DRB1 alleles were genotyped using the PCR with sequence specific primers (HLA DRB1 ${ }^{*}$ - PCR) supplied by Protrans and following manufacture's recommendations (PROTRANS Medicinische Diagnostische Produkte GmbH, Germany). Each samples were genotyped by a set of 24 PCRs, which resolved HLA DRB1 $1^{*}$, HLA DRB1 $1^{*} 03$, HLA DRB1 ${ }^{*} 04$, HLA DRB1 ${ }^{*} 07$, HLA DRB1 $1^{*} 08$, HLA DRB1 $^{*} 09$, HLA DRB1 ${ }^{*}$, HLA DRB $^{*} 12$, HLA DRB1 ${ }^{*} 13$, HLA DRB1 ${ }^{*} 14$, HLA DRB1* 15 , HLA DRB1 ${ }^{*} 16$. Laboratory analyses were carried out in the Laboratory of Clinical Chemistry and Genetics, Hospital of Lithuanian University of Health Sciences.

\section{Statistical analysis}

An analysis of the collected data was performed using the statistical package SPSS version 13.0. Comparisons of mean ages at onset of MS across groups were carried out using Student T- test. Parametric statistical criteria were used for the normally distributed quantitative variables (estimated with Kolmogorov-Smirnov and Shapiro-Wilk tests) and the mean and standard deviations (SD) of the mean were calculated. $\chi^{2}$ test was used to compare the qualitative variables and to estimate possible correlations. Odds ratios (OR) with $95 \%$ confidence interval (CI) were calculated looking for the associations. The determined level of significance $\alpha=0.05$, and $p$ values lower than 0.05 $(p<\alpha)$ were considered as statistically significant. Logistic regression models were constructed to assess immunological, genetic and clinical significance of signs of disease progression. A two-factor analysis was used to investigate two factors (genetic and immunological) effects of the observed random variable (disability) average.

\section{Results}

The MS group consisted of 120 subjects (44 (36.7\%) men and 76 (63.3\%) women). Mean age of MS patients was $43.75 \pm 10.1$ years (men $-41.79 \pm 9.97$ years, women $-44.8 \pm 10.05$ years). Of the $120 \mathrm{MS}$ patients, $50.0 \%$ had the relapsing-remitting (RR) course of the disease, $40.0 \%$, the secondary-progressive (SP) course, and $10.0 \%$, the primaryprogressive $(\mathrm{PP})$ course. Mean duration of symptoms was $11.93 \pm 8.0$ years (Table 1).

\begin{tabular}{|l|l|}
\hline \multirow{2}{*}{ Demographic and clinical characteristics } & MS patients \\
\cline { 2 - 2 } & $\mathbf{N}=\mathbf{1 2 0}$ \\
\hline Gender ratio (M:F) & $1: 1.72(44: 76)$ \\
\hline Age at onset, years, mean \pm SD (range) & $30.86 \pm 7.92(16-55)$ \\
\hline Disease course: & $60(50.0 \%)$ \\
\hline Relapsing-remitting & $48(40.0 \%)$ \\
\hline Secondary-progressive & $12(10.0 \%)$ \\
\hline Primary-progressive & $11.93 \pm 8.0$ \\
\hline Duration of the symptoms, years, mean, \pm SD & $6.84 \pm 3.54$ \\
\hline Duration of the disease (time of diagnosis), years, mean \pm SD & $1.36 \pm 0.88$ \\
\hline Relapse rate per year, mean \pm SD & $4.26 \pm 2.01$ \\
\hline EDSSA score, mean \pm SD & $3.8 \pm 1.0$ \\
\hline EDSSB score, mean \pm SD & $88(73.3 \%)$ \\
\hline Patients with positive OCBs (n, \%) & MS patients \\
\hline Demographic and clinical characteristics & \\
\hline
\end{tabular}


Citation: Balnyte R, Rastenyte D, Vaitkus A, Uloziene I, Vitkauskiene A, et al. (2015) Associations of HLA DRB1 Alleles with Igg Oligoclonal Bands and Their Influence on Multiple Sclerosis Course and Disability Status. 2155-9562 6: 273. doi:10.4172/2155-9562.1000273

Page 3 of 5

\begin{tabular}{|c|c|}
\hline & $N=120$ \\
\hline Gender ratio (M:F) & 1:1.72 (44:76) \\
\hline Age at onset, years, mean \pm SD (range) & $30.86 \pm 7.92(16-55)$ \\
\hline \multicolumn{2}{|l|}{ Disease course: } \\
\hline Relapsing-remitting & $60(50.0 \%)$ \\
\hline Secondary-progressive & $48(40.0 \%)$ \\
\hline Primary-progressive & $12(10.0 \%)$ \\
\hline Duration of the symptoms, years, mean, \pm SD & $11.93 \pm 8.0$ \\
\hline Duration of the disease (time of diagnosis), years, mean \pm SD & $6.84 \pm 3.54$ \\
\hline Relapse rate per year, mean \pm SD & $1.36 \pm 0.88$ \\
\hline EDSSA score, mean \pm SD & $4.26 \pm 2.01$ \\
\hline EDSSB score, mean \pm SD & $3.8 \pm 1.0$ \\
\hline Patients with positive OCBs (n, \%) & $88(73.3 \%)$ \\
\hline
\end{tabular}

Table 1: The main demographic and clinical characteristics of the multiple sclerosis (MS) patients

HLA DRB1 ${ }^{*} 08$ allele was more frequently documented among the RR MS patients than among the patients with the progressive forms of MS ( $25 \%$ vs. $8.3 \%$, respectively, $\mathrm{P}=0.014)$, while the HLA DRB1 ${ }^{*} 15$ allele was more prevalent among the patients with the progressive forms of MS (35\% vs. $20.8 \%$, respectively, $\mathrm{P}<0.001$ ) (data not shown). During the last visit, the lowest EDSS score was among the patients with the HLA DRB1 ${ }^{*} 08$ allele $(3.15 \pm 1.95)$, and the highest one among those with HLA DRB1*15 allele $(4.60 \pm 2.10)$ compared with the patients without these alleles $(4.49 \pm 1.96$ and $4.05 \pm 1.94$, respectively, $\mathrm{P}<0.05)$ but there were no significant associations between these alleles and the duration of the disease to disability (data not shown).

Positive OCBs were more prevalent among the MS patients with higher degree of disability ( $4.61 \pm 1.96$ vs. $3.31 \pm 1.89$, respectively, $\mathrm{P}=$ 0.002 ) and more frequent conversion to progressive course observed during the last visit ( $56.6 \%$ vs. $43.2 \%$, respectively, $\mathrm{P}=0.036$ ). The HLA $\mathrm{DRB} 1^{\star} 15$ allele was more common among the MS patients with OCBs in the CSF than among those without $(80.6 \%$ vs. $64.2 \%$; OR $=2.3,95 \%$ CI 1.017-5.301; $\mathrm{P}=0.043$ ) (data not shown).

HLA DRB1 ${ }^{*} 08$ allele demonstrated a relation of borderline significance to lower degree of disability, while no relation was found among other HLA DRB1 alleles and disability status, irrespective of the presence or absence of OCBs. Oligoclonal bands were an independent and significant factor that was related to disability status irrespectively of the HLA DRB1 ${ }^{*} 04,{ }^{*} 07,{ }^{*} 08,{ }^{* *}, 13,{ }^{*} 15$ and ${ }^{*} 16$ alleles. Disability status was also dependent on the interaction between HLA DRB1 ${ }^{*} 07$ allele and OCBs. No statistically significant associations were found between the other alleles and OCBs (Table 2).

\begin{tabular}{|l|l|l|l|}
\hline \multicolumn{2}{|l|}{ HLA DRB1 alleles value to disability } & OCB value to disability & $\begin{array}{l}\text { HLA DRB1 alleles and OCB interaction value } \\
\text { to disability }\end{array}$ \\
\hline Allele & $p$ value & $p$ value & $p$ value \\
\hline${ }^{*} 01$ & 0.2 & 0.7 & 0.38 \\
\hline${ }^{*} 03$ & 0.19 & 0.11 & 0.69 \\
\hline${ }^{*} 04$ & 0.087 & 0.008 & 0.92 \\
\hline${ }^{*} 07$ & 0.93 & 0.0001 & 0.049 \\
\hline$* 08$ & 0.062 & 0.018 & 0.87 \\
\hline$* 11$ & 0.14 & 0.005 & 0.41 \\
\hline$* 12$ & 0.11 & 0.14 & 0.51 \\
\hline$* 13$ & 0.18 & 0.005 & 0.83 \\
\hline
\end{tabular}


Citation: Balnyte R, Rastenyte D, Vaitkus A, Uloziene I, Vitkauskiene A, et al. (2015) Associations of HLA DRB1 Alleles with Igg Oligoclonal Bands and Their Influence on Multiple Sclerosis Course and Disability Status. 2155-9562 6: 273. doi:10.4172/2155-9562.1000273

Page 4 of 5

\begin{tabular}{|l|l|l|l|}
\hline$* 14$ & 0.32 & 0.7 & 0.13 \\
\hline$* 15$ & 0.4 & 0.003 & 0.73 \\
\hline$* 16$ & 0.23 & 0.024 & 0.3 \\
\hline
\end{tabular}

Table 2: Statistical significance of the HLA DRB1 alleles, oligoclonal bands, and their interactions to disability of multiple sclerosis patients (EDSS score), (two-factorial analysis; EDSS - Expanded Disability Status Scale, OCB - oligoclonal bands.

Logistic regression models were constructed in order to reveal an impact of age at onset, duration of symptoms, gender, HLA DRB1 alleles and oligoclonal bands on MS progression. Age at onset and duration of the disease were independent and significant factors for MS progression in all logistic regression models with each newly added HLA DRB1 allele (excluding models with ${ }^{*} 07,{ }^{* *} 13$, and ${ }^{*} 14$ alleles, where age at onset had only borderline significance). HLA
DRB $1{ }^{*} 08$ allele was related to $75 \%$ lower odds that RR MS will convert to progressive course MS irrespectively of other factors investigated (Table 3). In the model with ${ }^{*} 08$ allele, immunological factors had no impact on MS progression. Detection of oligoclonal bands in the CSF was associated with the higher possibility of RR MS conversion to a progressive course in all cases, except in the model with ${ }^{*} 08$ allele.

\begin{tabular}{|l|l|l|l|}
\hline Variable & $\boldsymbol{\beta}$ & OR (95\% Cl) & $\boldsymbol{p}$ value \\
\hline Age at onset & 0.072 & $1.07(1.00-1.15)$ & 0.046 \\
\hline Gender & 0.127 & $1.13(0.47-2.70)$ & 0.77 \\
\hline HLA DRB1*08 & 1.214 & $0.25(0.06-0.92)$ & 0.03 \\
\hline OCB & -1.649 & $1.37(0.61-3.08)$ & 0.4 \\
\hline IgG index & 0.893 & $2.41(0.31-18.9)$ & 0.39 \\
\hline Disease duration & 0.076 & $1.08(1.01-1.13)$ & 0.005 \\
\hline
\end{tabular}

Table 3: Odds to convert from relapsing remitting to progressive multiple sclerosis course (Logistic regression model with HLA DRB1 08 allele; $\beta$ (beta) - regression coefficient, OR - odds ratio, 95\% CI - confidence interval, OCB - oligoclonical bands, IgG index - immunoglobulin G index.

\section{Discussion}

Our study results suggest that OCB and HLA DRB1 alleles could influence both the natural course of MS and disability level. HLA DRB1 ${ }^{*} 08$ allele may be associated with a lower possibility that RR MS would convert to the progressive course of the disease regardless of other analyzed factors. Meanwhile, immunological factors (especially oligoclonal bands) were associated with the higher possibility of transition to the progressive course of the disease. They also influenced the progression of disability among people with MS Lithuania.

We found that HLA DRB1*08 allele tented to be associated with lower disability status and was more frequently among the RR MS, HLA DRB1 ${ }^{*} 15$ allele was more prevalent among the patients with the progressive forms of MS. Other HLA DRB1 alleles had no impact on disability level and disease course. Presence of OCBs in CSF in MS patients correlated with higher degree of disability and progressive course of the disease Oligoclonal bands were associated with disability status independently of HLA DRB1 ${ }^{*} 04,{ }^{*} 07,{ }^{*} 08,{ }^{*},{ }^{*} 13,{ }^{*} 15$ and ${ }^{*} 16$ alleles. Interaction between HLA DRB1 $1^{*} 07$ allele and OCBs had an impact on disability status. Age at onset of the first MS symptoms and disease duration were independent and significant factors for disease course to become progressive. HLA DRB1 ${ }^{\star} 08$ allele was associated with a $75 \%$ lower odds, that RR MS would change to progressive disease form independently of the other factors analysed. Furthermore, when this allele was detected, immunogenetic factors did not influence the change of disease course. Oligoclonal bands present in CSF increased the possibility for RR MS form to become progressive in all the cases analysed except when HLA DRB1 ${ }^{*} 08$ allele was detected. On the basis of this finding, it might be presumed, that OCBs could be considered as a marker related to the disease course conversion $[18,19,20]$ in MS.

Our findings reflected that immunogenetic factors, especially OCBs, are related to higher disability level, as well as to the tendency for disease course to become progressive, independent of the type of HLA DRB1 allele, except HLA DRB1 ${ }^{*} 08$ allele which was associated with better disease prognosis. According to the controversial and quite sparse data from literature, OCBs can be related to higher disability level, especially when HLA DRB1* 15 allele is detected, when HLA DRB $1^{\star} 04$ allele can be associated with higher disability level independently of OCBs presence [23]. Other authors claim that OCBs can determine better disability status [14]. Koch et al. found that there was no relation between OCBs and disability, as well as RR MS form tendency to change to SP form [18], in addition, L. Romero-Pinel et al. suggest that HLA DRB1 alleles have no impact on MS course change [24].

There is a lack of data evaluating how oligoclonal bands and HLA DRB1 gene alleles affect clinical manifestation of MS, furthermore they are controversial, therefore it is not possible to say which factors are the most significant for MS course and there is a strong need for larger studies in the future. According to our results, oligoclonal bands could 
be used to evaluate the clinical activity of disease and to forecast it's course, when the detection of HLA DRB1 gene alleles in the early stage of MS might be useful for foreseeing the clinical course and choosing the most appropriate prevention and management strategy in order to stop the progression of the disease and disability. However, detection of HLA DRB1 gene alleles in early stage of the disease should be studied more extensively in a larger patient group in order to predict the clinical course of the disease and choose the most appropriate prevention and management strategy thus reducing progression of the disease and disability.

Our study has several limitations: the data presented here were obtained from a relatively small MS patients group and this might influenced partly inconclusive results. So, this study should be judged as a pilot study and results have to be confirmed in the larger set of Lithuanian MS patients.

\section{Conclusions}

This study points to both distinct and shared genetic risk factors in Lithuanian MS patients differing by the presence or absence of OCB in CSF and reveals clinical differences between the groups. OCBs had an influence on disability status and disease course irrespective of HLA DRB1 alleles. HLA DRB1 ${ }^{*} 08$ allele was significantly associated with lower possibility that RR MS will change to progressive course MS, oligoclonal bands raised the possibility of disease progression. The HLA DRB1* 15 allele was found to be related to progressive course of the disease, and a higher degree of disability, while the HLA DRB $1^{\star} 08$ allele was more common in the patients with relapsing-remitting MS and a lower degree of disability. Our results suggest that OCBs and HLA DRB1 alleles may have an impact on the disability, natural course of the disease and thus provide with some additional information on prognosis and future perspectives to a patient; however, further and large-scale studies are needed.

\section{Acknowledgments}

The study was supported by the grant from the Research Foundation, Lithuanian University of Health Sciences.

\section{References}

1. Noseworthy JH, Lucchinetti C, Rodriguez M, Weinshenker BG (2000) Multiple sclerosis. N Engl J Med 343: 938-952.

2. Ramagopalan SV, Dobson R, Meier UC, Giovannoni G (2010) Multiple sclerosis: risk factors, prodromes, and potential causal pathways. Lancet Neurol 9: 727-739.

3. Oksenberg JR, Baranzini SE (2010) Multiple sclerosis genetics--is the glass half full, or half empty? Nat Rev Neurol 6: 429-437.

4. Ascherio A, Munger KL (2007) Environmental risk factors for multiple sclerosis. Part I: the role of infection. Ann Neurol 61: 288-299.

5. Ascherio A, Munger KL (2007) Environmental risk factors for multiple sclerosis. Part II: Noninfectious factors. Ann Neurol 61: 504-513.

6. Dyment DA, Ebers GC, Sadovnick AD (2004) Genetics of multiple sclerosis. Lancet Neurol 3: 104-110.
7. Ramagopalan SV, Dobson R, Meier UC, Giovannoni G (2010) Multiple sclerosis: risk factors, prodromes, and potential causal pathways. Lancet Neurol 9: 727-739.

8. Awad A, Hemmer B, Hartung HP, Kieseier B, Bennett JL, et al. (2010) Analyses of cerebrospinal fluid in the diagnosis and monitoring of multiple sclerosis. J Neuroimmunol 219: 1-7.

9. Freedman MS, Thompson EJ, Deisenhammer F, Giovannoni G, Grimsley $\mathrm{G}$, et al. (2005) Recommended standard of cerebrospinal fluid analysis in the diagnosis of multiple sclerosis: a consensus statement. Arch Neurol 62: 865-870.

10. Imrell K, Greiner E, Hillert J, Mastermann T (2009) HLA DRB1*15 and cerebrospinal-fluid-specific oligoclonal immunoglobulin $\mathrm{G}$ bands lower age at attainment of important disease milestones in multiple sclerosis. J Neuroimmunol 210:128-130.

11. Link H, Huang YM (2006) Oligoclonal bands in multiple sclerosis cerebrospinal fluid: an update on methodology and clinical usefulness. J Neuroimmunol 180: 17-28.

12. Romero-Pinel L, Martínez-Yélamos S, Bau L, Matas E, Gubieras L, et al. (2011) Association of HLA-DRB1* 15 allele and CSF oligoclonal bands in a Spanish multiple sclerosis cohort. Eur J Neurol 18: 1258-1262.

13. Gama PD, Machado Ldos R, Livramento JA, Gomes HR, Adoni T, et al. (2009) Study of oligoclonal bands restricted to the cerebrospinal fluid in multiple sclerosis patients in the city of São Paulo. Arq Neuropsiquiatr 67: 1017-1022.

14. Idiman E, Ozakbas S, Dogan Y, Kosehasanogullari G (2009) The significance of oligoclonal bands in multiple sclerosis: relevance of demographic and clinical features, and immunogenetic backgrounds. J Neuroimmunol 212: 121-124.

15. Nakashima I, Fujihara K, Misu T, Fujimori J, Sato S, et al. (2002) A comparative study of Japanese multiple sclerosis patients with and without oligoclonal IgG bands. Mult Scler 8: 459-462.

16. Wu JS, Qiu W, Castley A, James I, Joseph J, et al. (2010) Presence of CSF oligoclonal bands (OCB) is associated with the HLA-DRB1 genotype in a West Australian multiple sclerosis cohort. J Neurol Sci 288: 63-67.

17. Fredrikson S (1994) CSF - abnormalities and multipe sclerosis diagnosis. Int MS J 1: 69-71.

18. Koch M, Heersema D, Mostert J, Teelken A, De Keyser J (2007) Cerebrospinal fluid oligoclonal bands and progression of disability in multiple sclerosis. Eur J Neurol 14: 797-800.

19. Siritho S, Freedman MS (2009) The prognostic significance of cerebrospinal fluid in multiple sclerosis. J Neurol Sci 279: 21-25.

20. Amato MP, Ponziani G (2000) A prospective study on the prognosis of multiple sclerosis. Neurol Sci 21: S831-838.

21. Sá MJ, Sequeira L, Rio ME, Thompson EJ (2005) [Oligoclonal IgG bands in the cerebrospinal fluid of portuguese patients with multiple sclerosis: negative results indicate benign disease]. Arq Neuropsiquiatr 63: 375-379.

22. Barcellos LF, Sawcer S, Ramsay PP, Baranzini SE, Thomson G, et al. (2006) Heterogeneity at the HLA-DRB1 locus and risk for multiple sclerosis. Hum Mol Genet 15: 2813-2824.

23. Hemmer B, Nessler S, Zhou D, Kieseier B, Hartung HP (2006) Immunopathogenesis and immunotherapy of multiple sclerosis. Nat Clin Pract Neurol 2: 201-211.

24. Romero-Pinel L, Pujal JM, Martínez-Yélamos S, Gubieras L, Matas E, et al. (2011) HLA-DRB1: genetic susceptibility and disability progression in a Spanish multiple sclerosis population. Eur J Neurol 18: 337-342. 